DOI: https://doi.org/10.22206/ceyn.2021.v5i1.pp119-137

\title{
Costos Educativos del Covid 19 con y sin Optimización de Presencialidad: una estimación del caso de República Dominicana
}

\author{
Rolando ReYes ${ }^{\mathrm{a}}$
}

Recibido: 2/3/2021 • Aprobado: 20/5/2021

Cómo citar: Reyes, R. (2021). Costos Educativos del Covid 19 con y sin Optimización de Presencialidad: una estimación del caso de República Dominicana. Ciencia, Economía y Negocios, 5(1), 119-137. Doi: https:// doi.org/10.22206/ceyn.2021.v5i1.pp119-137

\section{Resumen}

Se formula y se aplica un modelo que permite cuantificar las pérdidas del cierre escolar inducido por la pandemia del Covid 19 en dos escenarios, partiendo del mismo nivel de ganancia de aprendizaje esperado en un año escolar normal. En el primero, el porcentaje de no presencialidad es sustituido en cien por ciento por la educación a distancia, y en el segundo se utiliza el modelo que minimiza el costo educativo pandémico total, representado por los costos del contagio de la presencialidad y los costos de las pérdidas de aprendizajes de los distintos medios no presenciales. La metodología aplicada en el primer escenario se asume un cierre equivalente al $80 \%$ del tiempo del año escolar, y un efecto adicional de ausencia escolar por pérdidas de ingresos de los hogares de los alumnos. En el segundo escenario se calcula la combinación óptima de presencialidad y no presencialidad, y luego se repiten los cálculos de las pérdidas de aprendizaje. Cuando se considera el promedio del valor de las pérdidas de aprendizajes de las cinco opciones no presenciales, calculado en el escenario sin combinación óptima, y se repite dicho cálculo para el caso de dicha combinación, se concluye que usando esta última, las autoridades educativas hubiesen evitado unas pérdidas de aprendizaje equivalente al $3.89 \%$ del PIB.

Palabras clave: docencia virtual y presencial; pérdidas y costos de aprendizaje; optimización de modalidades educativas; impactos educativos del Covid 19.

Códigos del JEL.: I24, I25, I31, J24

a Universidad Ambiental Fernando Arturo de Meriño, Jarabacoa, República Dominicana. ORCID: 0000-0001-7160-5961, Correo-e: rectoria@uafam.edu.do

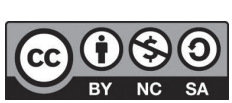

Esta obra está bajo licencia internacional Creative Commons Atribución-NoComercial-CompartirIgual 4.0 Internacional 


\title{
Covid 19 Educational Costs with and Without Presence Optimization: An estimate of THE DoMinican Republic case
}

\section{ROLANDO REYES ${ }^{\mathrm{a}}$}

\author{
Received: 2/3/2021 - Approved: 20/5/2021
}

\begin{abstract}
This paper presents a model that allows quantifying the cost of school closure during the Covid-19 pandemic. There are two main scenarios: the first one assumes $100 \%$ virtual education. The second scenario uses a model that minimizes the total pandemic cost, weighing the cost of contagion and the cost of learning losses from different non-face-to-face educational methods. The results show that, when considering the average value of learning losses from five different methods of distant education, educational authorities would have avoided learning losses equivalent to 3.89\% of GDP.
\end{abstract}

Keywords: Virtual and face-to-face teaching; Losses and learning costs; optimization of educational modalities; Educational impacts of Covid 19.

JEL codes: I24, I25, I3I

a Universidad Ambiental Fernando Arturo de Merińo, Jarabacoa, Dominican Republic. ORCID: 0000-0001-7160-5961, E-mail: rectoria@uafam.edu.do 


\section{Introducción}

La pandemia del Covid 19 ha tenido un triple efecto negativo sobre los sistemas educativos. El primero ha sido el impacto directo que significa el cierre de los centros de educación, con lo cual se ha buscado evitar el contacto entre alumnos, docentes y terceros. El segundo ha sido un impacto indirecto a través de la caída en los ingresos de los hogares, lo cual disminuye la capacidad de gasto en educación; mientras que el tercero y último es el resultado combinado de los dos anteriores: una disminución de la cantidad y calidad de los aprendizajes que se lograrían durante el año escolar pandémico.

Este impacto sería mayor en los países de bajos y medianos ingresos, debido a que en los mismos los medios de acceso a una educación a distancia son más escasos, la intensidad del choque negativo sobre los ingresos de los hogares podría ser mucho más significativo que en los países de altos ingresos y, sobre todo, debido al mayor retraso educativo relativo que presenta el conjunto de estos países.

Muchos países se vieron obligados a posponer la apertura de los centros educativos y a aplicar modelos de educación no presencial hasta registrar una reducción significativa de la incidencia de la pandemia, sobre todo mediante la vacunación masiva de su población escolar. La utilización de medios no presenciales reduce los costos del contagio de la pandemia, pero aumenta los costos de pérdidas de aprendizaje de los medios no presenciales. La suma de ambos costos se podría denominar como Costos Educativos del Covid 19.

El cierre de centros educativos y la utilización de medios remotos reducen la cantidad y calidad de los aprendizajes, lo que produce un segundo costo, representado por el valor presente de los ingresos futuros que los alumnos actuales perderían a su entrada al mercado laboral, lo que a su vez se explica por su menor productividad o capital humano. A este costo se le podría denominar Costo por Pérdida de Aprendizaje. La suma del "Costo del Contagio" y del "Costo de Pérdida de Aprendizaje" es el "Costo Total del Covid 19", y el propósito fundamental de este artículo es presentar un modelo y una metodología para la cuantificación del mismo, aplicados al caso de la Republica Dominicana. 
El resto del artículo está organizado de la siguiente manera: en la sección que sigue se presenta una breve discusión de literatura más relevante y directamente vinculada a la efectividad de las distintas modalidades educativas y al impacto de la calidad de la escolaridad en la productividad del capital humano. En la segunda sección se presenta un modelo de optimización de medios o modalidades educativas que minimiza el costo total de contagio de la pandemia, el cual arroja como uno de sus resultados la fracción del año escolar que debería impartirse a través de medios remotos o no presenciales.

El cierre y el componente remoto tienen el costo de la reducción de los aprendizajes, el cual puede reducirse a través del acompańamiento al alumno y a los padres, lo que a su vez genera un nuevo costo. La suma de ambos costos se minimiza en el punto en el que los beneficios de la reducción de las pérdidas de aprendizaje se igualan a los costos del acompańamiento. Este componente del modelo se presenta en la última parte de la segunda sección. La tercera y última parte presenta y discute los resultados de la aplicación del modelo al caso de la República Dominicana, y expone un resumen de las principales conclusiones a que conducen dichos resultados.

\section{Breve revisión de la principal literatura relacionada directamente con el costo educativo total de la pandemia}

Los costos de la modalidad presencial, en términos de riesgos de contagio de la pandemia, versus los costos de implementar la modalidad no presencial es un tema completamente ausente en toda la literatura que se ha generado sobre los efectos del Covid 19 en los sistemas educativos de todos los países del mundo.

Bettinger et al. (2020) consideran la modalidad no presencial como factor de producción de servicios educativos. Estos autores estiman una función de producción educativa mediante el uso de un ensayo que varía la dosis de aprendizaje asistido por computadora (CAL) como sustituto del aprendizaje tradicional. Los resultados muestran que la producción es cóncava con respecto al CAL. Pasando de cero a un nivel bajo de CAL, la tasa marginal de sustitución técnica (MRTS) de CAL para el aprendizaje tradicional es mayor que uno. Pasando de un nivel de CAL inferior a 
otro superior, la producción permanece en la misma, o en una isocuanta más baja, y la MRTS es igual o menor que uno. Los autores concluyen que las estimaciones son consistentes con la forma general de una función de producción Cobb-Douglas e implican que un enfoque combinado de CAL y aprendizaje tradicional es óptimo.

Lo más importante a destacar del marco analítico utilizado en el artículo citado es que existe una combinación o una tasa de sustitución entre las dos modalidades o insumos que mantiene constante la producción de aprendizaje en el alumno, y desde el punto de vista del enfoque del modelo que se presenta más adelante, su implicación es la existencia de una combinación óptima de modalidades tradicional y asistida por computadora.

Sin embargo, a diferencia del enfoque de la curva de una isocuanta que mantiene el nivel de aprendizaje constante, y en el que la optimización se produce con respecto a los costos de las modalidades que produce el mismo nivel de aprendizaje, en nuestro enfoque la optimización se produce con respecto a los costos totales de la pandemia, en el que en una primera etapa se minimiza el costo pandémico total de la combinación de modalidades educativas presencial y no presencial de cada centro o comunidad educativa, mientras que en una segunda etapa se minimiza el costo total de la pérdida de aprendizaje por parte de los alumnos sujetos a los requisitos del control de la pandemia.

En cambio, la literatura sobre los impactos de las modalidades no presenciales y de la reducción de los tiempos de instrucción en los logros educativos y los aprendizajes de los alumnos, sí tiene algunas referencias relacionadas directamente al tema de este trabajo. Una revisión de las evidencias de los usos e impactos de las tecnologías educativas se puede encontrar en Escueta, Quan, Nickow y Oreopoulos (2017), en la cual se clasifican las mismas en cuatro categorías: (1) acceso a la tecnología, (2) aprendizaje asistido por computadora, (3) intervenciones conductuales habilitadas por la tecnología en la educación y (4) aprendizaje en línea.

Un importante estudio que se centra en los impactos del uso de la tecnología digital en los logros de la educación se encuentra en Education Endowment Foundation (2019). cuyos autores, con base en la revisión documental realizada, concluyen: 
... que la tecnología digital está asociada con ganancias de aprendizaje moderadas: en promedio, cuatro meses adicionales de progreso. Sin embargo, existe una variación considerable en el impacto. La evidencia sugiere que los enfoques tecnológicos deberían usarse para complementar otras enseñanzas, en lugar de reemplazar enfoques más tradicionales (p. 1).

Perera y Aboal (2019) presentan una investigación del impacto del uso de una plataforma de adaptación para el estudio de matemática en el Perú. El estudio muestra que el uso de dicha plataforma produce un aumento de 0.2 desviaciones estándar en las pruebas de matemáticas de los alumnos de la educación primaria. También se encontró que el impacto del uso de la plataforma es mayor en los alumnos de menor nivel socioeconómico, lo cual es un sorprendente resultado de esta investigación.

Un importante estudio del impacto de los tiempos de instrucción en los aprendizajes de los alumnos se evidencia en Cattaneo, Oggenfuss y Wolter (2016); entre los principales hallazgos de este estudio se encuentra:

... un impacto significativo del tiempo de instrucción adicional en los resultados del aprendizaje medidos con los puntajes de las pruebas PISA, pero la efectividad de una hora adicional de instrucción es solo entre el treinta y el cuarenta por ciento del impacto que esperaríamos de una hora promedio de instrucción. En otras palabras, las autoridades educativas deben considerar cuidadosamente las variaciones del tiempo de instrucción, ya que las ganancias marginales de más tiempo de instrucción pueden ser demasiado bajas en comparación con los usos alternativos del tiempo y los recursos financieros necesarios para el tiempo de instrucción adicional (pp. 9-10).

Las pérdidas de aprendizaje que puede producir la interrupción del periodo escolar por el cierre de centros educativos impuesto por la pandemia puede ser comprendido a partir del estudio de Christopher Cummiskey y Jonathan Stern (2020). En forma simple y resumida, la principal implicación del citado estudio es que las pérdidas de aprendizaje causado por la disminución total o parcial del periodo escolar se puede pronosticar en 
función de la diferencia de puntajes entre los alumnos de dos grados sucesivos. La importancia de este resultado radica en el hecho de que, en una situación de pérdida parcial del periodo escolar de un grado cualquiera, la disminución de aprendizaje que se produciría se podría estimar aplicando el porcentaje de disminución del periodo de instrucción a la diferencia de aprendizaje del siguiente grado.

Otra clasificación de la literatura pertinente y relevante al tema de este artículo es la que estudia y analiza los costos de las pérdidas de aprendizaje de los alumnos, en este caso, la que están determinadas por el cierre, la modalidad no presencial y la reducción de los tiempos de instrucción de la Pandemia del Covid 19, y por supuesto, la literatura sobre este tema es extremadamente escasa, pero quizás el más importante y de mayor influencia sea el del Banco Mundial (2020), y en el cual se fundamenta una parte del enfoque metodológico y analítico seguido en este artículo.

\section{El Modelo de Minimización de los Costos Educativos Totales del Covid 19}

Se parte de dos tipos de costos, los cuales se generan en dos momentos diferentes: los costos de contagio en que se incurriría al momento de la apertura de los centros educativos (los cuales deben ser considerados y analizados al momento de decidir la apertura de los mismos), y los costos de pérdidas de aprendizaje, en los cuales se incurre durante el cierre, debido a la ineficiencia de las modalidad no presencial, cuya productividad educativa, en términos de aprendizaje obtenido entre grados sucesivos (Cummiskey y Stern, 2020), es menor que la presencial.

El punto de partida del proceso de determinación del primer tipo de costo es la incidencia del contacto personal en la trasmisión del virus, la cual aumenta con la presencialidad de los alumnos y el resto de la población escolar en los centros educativos. El costo del contagio de la pandemia, medidos en términos de años de vida estadística perdidos, es mayor que el costo esperado pandémico total (Reyes, 2020), ya que la edad promedio de la población escolar es menor que la edad promedio de la población total. 
El costo educativo del contagio de la pandemia se reduce con la virtualidad. Pero a mayor virtualidad, mayor costo de los medios de la educación a distancia medidos en términos de pérdidas de aprendizaje. Por tanto, si el costo educativo del contagio aumenta con la presencialidad, mientras que lo inverso ocurre con el costo de la virtualidad, entonces siempre existirá un punto óptimo en el que suma de ambos costos se minimiza.

El tiempo acumulado del cierre, y la utilización de medios o modalidades no presenciales generan una pérdida de aprendizaje con relación a la situación normal de completa presencialidad del año escolar. Una vez decidida la apertura con el grado de presencialidad determinada según la minimización de costos educativos del Covid 19 anteriormente expuesta, se determina un nivel de aprendizaje correspondiente a la combinación óptima, y la diferencia de este con respecto al aprendizaje obtenido con la modalidad a distancia utilizada durante el cierre, sería el costo de no elegir o decidir la apertura con el grado de presencialidad óptima.

El costo por pérdida de aprendizaje tiene dos características fundamentales: es inversamente proporcional a la efectividad de los medios no presenciales utilizados, y se puede mitigar a través de instrumentos y medidas de acompańamiento a los alumnos, tal y como lo demuestran Muralidharan, Singh y Ganimian (2019), quienes encontraron que asistir a un programa extracurricular durante 90 días puede aumentar el puntaje en matemática en 0.6 desviaciones estándar.

Los costos por pérdidas de aprendizaje disminuyen con la efectividad de los medios no presenciales, y para aumentar esta última se incurre en un costo de mitigación, que es proporcional a la misma, por lo que el costo total se minimiza en el punto que ambos costos se igualan, lo cual constituye el segundo y último momento de minimización de los costos totales educativos del Covid 19.

La figura 1 muestra el mapa conceptual correspondiente al modelo anteriormente expuesto, en la que se pueden observar la relación causaefecto de cada una de las variables. 

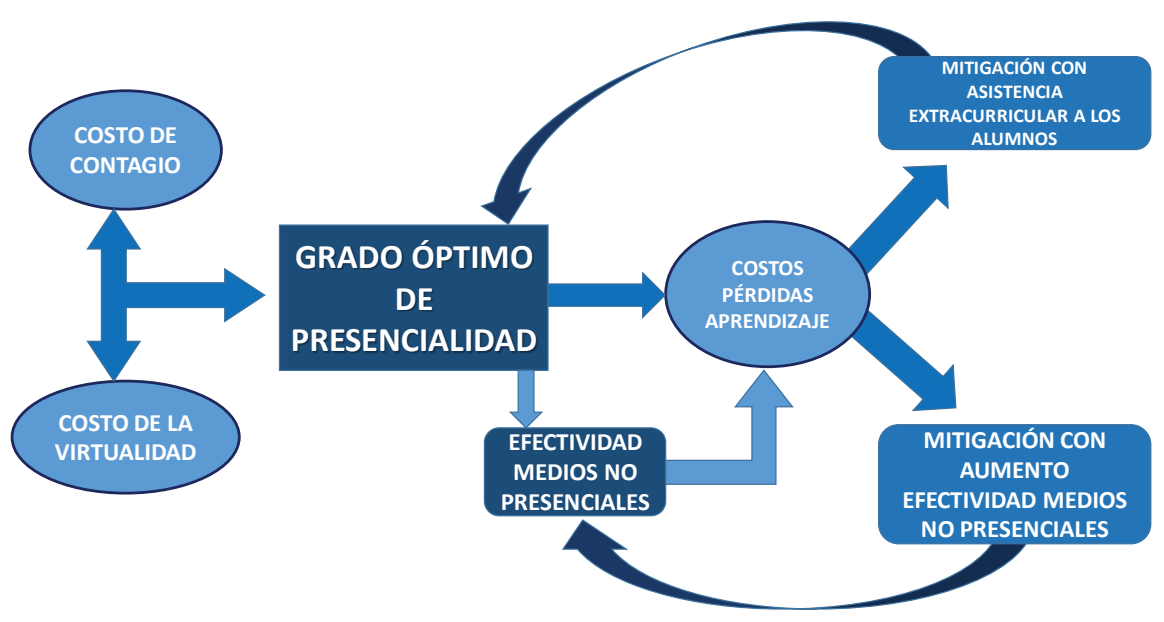

Figura 1. Mapa Conceptual del Modelo de Minimización del Costo Educativo Total del Covid 19 en los Centros Educativos

Fuente: elaboración propia.

La combinación óptima de modalidades que minimiza el costo total pandémico del primer momento genera una pérdida de aprendizaje, que es la diferencia entre el nivel de aprendizaje esperado y el que se hubiese obtenido en la presencialidad de la normalidad.

Dado el nivel de presencialidad, la pérdida de aprendizaje depende de la efectividad de los medios no presenciales, por lo que la misma se puede mitigar con acciones que aumenten la efectividad de dichos medios, tales como el acompañamiento del alumno, el aumento del acceso a dispositivos y conectividad, y el suministro de material de apoyo complementario.

La pérdida de aprendizaje también se puede mitigar mediante la asistencia extracurricular a los alumnos, la cual estaría condicionada por el grado de presencialidad óptimo previamente calculado, el cual debe mantenerse hasta que la incidencia de la pandemia se reduzca al nivel en que pueda cambiarse la combinación óptima de modalidades del primer momento.

El proceso de determinación del costo educativo total del Covid 19 se ilustra en el conjunto de gráficos que contiene la figura 2, en la que P es 
el nivel de Presencialidad, C el Costo de ambas modalidades, L el Aprendizaje, y E la Efectividad de cada modalidad no presencial en lograr el nivel de aprendizaje esperado.

Tal y como se puede observar en los dos gráficos del lado izquierdo, en el primer momento la igualación del Costo del Contagio (CC) con el Costo de la Virtualidad (CV), medido en términos de Costos de Pérdidas de Aprendizaje (CPA), se determina el grado óptimo de presencialidad (gráfico superior del lado izquierdo, o panel 1). Dado que el Aprendizaje, L, es una función creciente del grado de Presencialidad, su nivel queda determinado por el grado óptimo de esta última (gráfico inferior del lado izquierdo, o panel 2)

La variable fundamental del modelo es el Costo de la Pérdida de Aprendizaje (CPA), el cual aumenta con la disminución de dicho Aprendizaje, tal y como se refleja en el lado inferior del lado derecho o panel 3 de la figura 2. Pero el CPA depende de la Efectividad (E) de cada modalidad, y mientras mayor sea esta última, menor será el primero. Pero la Efectividad se puede aumentar mediante medidas que generan un Costo de Mitigación (CM). La comparación del CM y el CPA es la clave para el segundo momento o segunda optimización del costo total de ambas modalidades. Siempre se debe esperar que el Costo de la Pérdida de Aprendizaje sea mayor que el Costo de la Mitigación, pues al principio este último no existe o es muy bajo. La distancia entre ambos que ilustra el gráfico superior del lado derecho representa la oportunidad de invertir de manera altamente rentable en la disminución de las pérdidas de aprendizaje.

El gráfico superior del lado derecho o panel 4 muestra que la modalidad inicial de combinación óptima produce un CPA igual a a2, el cual corresponde a un nivel de Efectividad muy bajo, el cual tiene un CM de a1, mucho menor que a2. Utilizando los medios de mitigación adecuados, los centros educativos del sistema pueden incurrir en aumentos de CM que producirían aumentos de Aprendizajes (reducirían los CPA) a un costo siempre menor al aumento del CM, optimizando nuevamente el costo educativo total de la pandemia del Covid 19, hasta el punto en el que el CM y el CPA se igualan, tal y como lo representa la recta color naranja. 


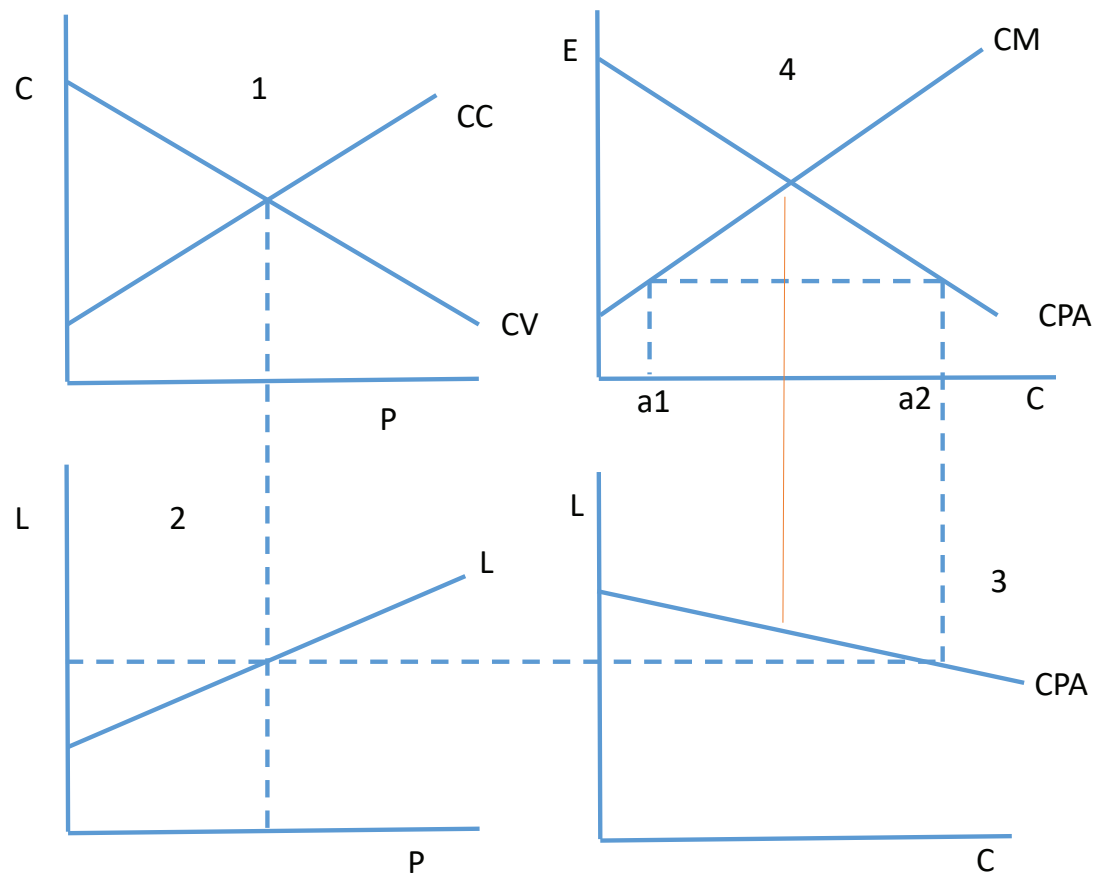

Figura 2. Representación gráfica del proceso completo de Minimización del Costo Educativo Total del Covid 19

Fuente: elaboración propia.

\section{Costo total del Covid 19 en el Sistema Educativo Dominicano}

Se ha estimado el Costo Total de la Pandemia en el Sistema Educativo Dominicano en dos escenarios. En el primero se utiliza un porcentaje del $20 \%$ del tiempo del año escolar en presencialidad, mientras que en el segundo se utiliza la combinación óptima de modalidades calculada con el modelo presentado con base en una muestra que representa el $90 \%$ de los centros educativos del Distrito Escolar del municipio de Jarabacoa.

Se ha estimado que en un ańo escolar normal un alumno promedio del sistema educativo obtiene una ganancia de aprendizaje de 30 puntos, medidos según los criterios internacionales referidos y utilizados por el 
Banco Mundial (2020). En ambos escenarios se utilizan cinco medios no presenciales con grado de efectividad creciente en el siguiente orden: radio y televisión sin mitigación; radio y televisión con mitigación; internet con baja accesibilidad; internet con alta accesibilidad; e internet con plataforma digital.

Siguiendo los criterios del Banco Mundial (World Bank Group, 2020) se ha calculado el grado de efectividad de cada uno de los medios no presenciales como el producto de la cobertura de la provisión del gobierno para el aprendizaje no presencial $(\mathrm{G})$, el grado de acceso de los alumnos a los medios no presenciales (A), y la efectividad de cada uno de los medios no presenciales (E).

La pérdida de aprendizaje es el producto de la ganancia esperada del mismo, el porcentaje de presencialidad asumido, y la inefectividad del medio no presencial utilizado, último elemento de la metodología del Banco Mundial (2020) utilizado en este trabajo. El cuadro 1, que sigue a continuación, presenta los cálculos sin optimización de la combinación de modalidades, es decir, asumiendo que los centros educativos estarán cerrados durante el $80 \%$ del año escolar. El primer paso del proceso de cuantificación es el cálculo de la Efectividad de cada medio no presencial, para lo cual se aplica la formula señalada en el párrafo anterior, y cuyos resultados se presentan en la tercera línea del citado cuadro.

El segundo paso consiste en el cálculo de la pérdida de aprendizaje que hace posible el dato de la efectividad de cada medio. Como se puede observar, el caso del medio menos efectivo (radio y TV sin mitigación) se perdería un poco más de la mitad de los aprendizajes que se esperarían ganar durante el año escolar, mientras con el medio más efectivo (internet de alta conectividad con plataforma electrónica), la pérdida baja a un $30 \%$.

Además de las pérdidas por cierre de los centros educativos, también se consideran las pérdidas por inasistencia escolar, que causa las reducciones de ingresos de los hogares que provoca la crisis pandémica. Nuestra estimación de este efecto se calcula como el producto del Aprendizaje esperado, el porcentaje de disminución de los ingresos de los hogares, y la elasticidad asistencia escolar/ingreso del hogar. Con una reducción uniforme del $5 \%$ de los ingresos de los hogares y una elasticidad de 0.9 , la pérdida de apren- 
dizaje en todos los medios no presenciales del cuadro 1 es del $4.5 \%$.

Como se puede observar en el cuadro 1 , en el caso del medio menos efectivo, la pérdida total de aprendizaje es de 16.43 puntos, equivalente a una pérdida del $55 \%$ del año escolar, mientras que, en el caso del medio más efectivo, la pérdida equivale a $34 \%$ del año escolar.

Cuadro 1. Costo Total Pérdidas de Aprendizaje Causadas por el Covid sin Optimización Modalidades, y según Efectividad Medios no Presenciales

\begin{tabular}{|l|c|c|c|c|c|}
\hline \multicolumn{1}{|c|}{ Detalle } & $\begin{array}{c}\text { Radio y } \\
\text { TV, SM }\end{array}$ & $\begin{array}{c}\text { Radio y } \\
\text { TV, CM }\end{array}$ & $\begin{array}{c}\text { Internet } \\
\text { bajo }\end{array}$ & $\begin{array}{c}\text { Internet } \\
\text { alto }\end{array}$ & $\begin{array}{c}\text { Internet } \\
\text { con } \\
\text { Plataforma }\end{array}$ \\
\hline $\begin{array}{l}\text { Ganancias de } \\
\text { aprendizaje } \\
\text { año escolar en } \\
\text { Normalidad }\end{array}$ & 30.00 & 30.00 & 30.00 & 30.00 & 30.00 \\
\hline $\begin{array}{l}\text { \% Ańo escolar en no } \\
\text { Presencialidad }\end{array}$ & 0.80 & 0.80 & 0.80 & 0.80 & 0.80 \\
\hline $\begin{array}{l}\text { Efectividad } \\
\text { componente no } \\
\text { presencial }\end{array}$ & 0.162 & 0.243 & 0.294 & 0.392 & 0.504 \\
\hline $\begin{array}{l}\text { Pérdidas de } \\
\text { aprendizaje por } \\
\text { Cierre }\end{array}$ & 20.11 & 18.17 & 16.94 & 14.59 & 11.90 \\
\hline $\begin{array}{l}\text { Elasticidad ingreso } \\
\text { inasistencia escolar }\end{array}$ & 0.90 & 0.90 & 0.90 & 0.90 & 0.90 \\
\hline $\begin{array}{l}\text { Disminución del } \\
\text { ingreso del hogar }\end{array}$ & 0.05 & 0.05 & 0.05 & 0.05 & 0.05 \\
\hline $\begin{array}{l}\text { Pérdidas de } \\
\text { aprendizaje } \\
\text { inasistencia por } \\
\text { pérdida Ingreso }\end{array}$ & 1.35 & 1.35 & 1.35 & 1.35 & 1.35 \\
\hline $\begin{array}{l}\text { Pérdida total de } \\
\text { aprendizaje }\end{array}$ & 21.46 & 19.52 & 18.29 & 15.94 & 13.25 \\
\hline $\begin{array}{l}\text { Pérdida de } \\
\text { aprendizaje como \% } \\
\text { año escolar }\end{array}$ & 0.72 & 0.65 & 0.61 & 0.53 & 0.44 \\
\hline $\begin{array}{l}\text { Ańos de escolaridad } \\
\text { esperado de 4 a 18 } \\
\text { años }\end{array}$ & 11.30 & 11.30 & 11.30 & 11.30 & 11.30 \\
\hline
\end{tabular}




\begin{tabular}{|l|c|c|c|c|c|}
\hline \multicolumn{1}{|c|}{ Detalle } & $\begin{array}{c}\text { Radio y } \\
\text { TV, SM }\end{array}$ & $\begin{array}{c}\text { Radio y } \\
\text { TV, CM }\end{array}$ & $\begin{array}{c}\text { Internet } \\
\text { bajo }\end{array}$ & $\begin{array}{c}\text { Internet } \\
\text { alto }\end{array}$ & $\begin{array}{c}\text { Internet } \\
\text { con } \\
\text { Plataforma }\end{array}$ \\
\hline $\begin{array}{l}\text { Ańos de escolaridad } \\
\text { ajustados por } \\
\text { aprendizaje }\end{array}$ & 6.30 & 6.30 & 6.30 & 6.30 & 6.30 \\
\hline $\begin{array}{l}\text { Años de escolaridad } \\
\text { sin optimización } \\
\text { modalidades }\end{array}$ & 5.58 & 5.65 & 5.69 & 5.77 & 5.86 \\
\hline $\begin{array}{l}\text { Ganancias salariales } \\
\text { en US\$ corriente }\end{array}$ & $4,117.33$ & $4,117.33$ & $4,117.33$ & $4,117.33$ & $4,117.33$ \\
\hline $\begin{array}{l}\text { Pérdidas por alumno } \\
\text { por reducción } \\
\text { aprendizaje, US\$ }\end{array}$ & 235.64 & 214.30 & 200.86 & 175.04 & 145.52 \\
\hline $\begin{array}{l}\text { Valor presente } \\
\text { pérdidas de } \\
\text { escolaridad por } \\
\text { alumno, US\$ }\end{array}$ & $4,188.34$ & $3,808.96$ & $3,570.10$ & $3,111.10$ & $2,586.54$ \\
\hline $\begin{array}{l}\text { Pérdida Sistema } \\
\text { Educativo no } \\
\text { universitario, MM } \\
\text { de US\$ }\end{array}$ & $10,052.01$ & $9,141.52$ & $8,568.24$ & $7,466.65$ & $6,207.69$ \\
\hline $\begin{array}{l}\text { Pérdida como \% del } \\
\text { PIB }\end{array}$ & $12.77 \%$ & $11.62 \%$ & $10.89 \%$ & $9.49 \%$ & $7.89 \%$ \\
\hline $\begin{array}{l}\text { Pérdida como \% } \\
\text { del Presupuesto } \\
\text { MINERD }\end{array}$ & $51.1 \%$ & $46.5 \%$ & $43.6 \%$ & $38.0 \%$ & $31.6 \%$ \\
\hline
\end{tabular}

Fuente: elaboración propia.

El tercer y último gran paso de la metodología del cálculo del modelo es la monetización de las pérdidas de aprendizaje. En términos generales, la pérdida monetaria por reducción del aprendizaje registrada durante el año escolar de la pandemia es el valor presente de los ingresos o ganancias salariales dejados de percibir por cada uno de los miembros de la cohorte de alumnos durante el resto de toda su vida laboral.

Con una ganancia salarial anual promedio de US\$ 4.117 .33 y una tasa de rentabilidad de la inversión privada en educación del 8 \% (Banco 
Mundial, 2020), la pérdida anual por alumno utilizando el medio no presencial menos efectivo sería de US\$235.64, mientras que en el caso de que se utilizase el más efectivo sería de US\$ 145.52. Usando una tasa de descuento del $5 \%$, y una vida laboral promedio de 45 años, el valor presente de las pérdidas de los alumnos usando los medios menos eficientes es de US\$ 4,188.34, bajando a tan solo US\$2,586.54 en los alumnos con acceso a los medios más eficientes.

Asumiendo 2,400,000 alumnos, las pérdidas totales si todos estos utilizaran el medio no presencial menos eficiente sería de US\$ 10,052.1 millones de dólares, equivalente al $12.77 \%$ del PIB y al $51.1 \%$ del presupuesto del Ministerio de Educación. En el caso de que todos los alumnos utilizaran el medio más eficiente, estas cifras bajaría a US\$ 6,207.69 millones de dólares, $7.89 \%$ del PIB y $31.6 \%$ del presupuesto del ministerio.

El cuadro 2, que sigue a continuación, presenta los mismos cálculos que el cuadro 1, con la gran diferencia de que ahora el porcentaje no presencial del año escolar ha sido determinado utilizando las ecuaciones que representan las curvas del panel 1 del gráfico 2, utilizando una muestra de 74 centros educativos del Distrito Escolar 0603 del Municipio de Jarabacoa. La no presencialidad baja de un $80 \%$ a un $48 \%$, lo que produce el efecto inmediato y directo de reducir las pérdidas de aprendizaje, lo que a su vez cambia todos los valores monetarios de dichas pérdidas, representados en las últimas cinco líneas del citado cuadro.

El resumen del impacto de este cambio en los grupos de menores ingresos y, por ende, con acceso mayoritario a los medios virtuales menos eficientes (radio y TV, sin mitigación), se puede resumir de la siguiente manera:

- El valor presente de las pérdidas por aprendizaje se reduce en US\$ 1569.95 anuales

- La pérdida del sistema educativo (si todos los alumnos utilizaron el medio menos eficiente) se reduciría en US\$ 3,767.89 millones

- Las pérdidas como \% del PIB bajarían de $12.77 \%$ a $7.99 \%$

- Las pérdidas como \% del presupuesto del ministerio bajarían de $51.1 \%$ a $31.9 \%$ 
Cuadro 2. Costo Total Pérdidas de Aprendizaje causadas por el Covid sin Optimización Modalidades, y según Efectividad Medios no Presenciales

\begin{tabular}{|c|c|c|c|c|c|}
\hline Detalle & $\begin{array}{l}\text { Radio y } \\
\text { TV, SM }\end{array}$ & $\begin{array}{l}\text { Radio y } \\
\text { TV, CM }\end{array}$ & $\begin{array}{l}\text { Internet } \\
\text { bajo }\end{array}$ & $\begin{array}{l}\text { Internet } \\
\text { alto }\end{array}$ & $\begin{array}{l}\text { Internet } \\
\text { con Plat }\end{array}$ \\
\hline $\begin{array}{l}\text { Ganancias de aprendizaje } \\
\text { año escolar en Normalidad }\end{array}$ & 30.00 & 30.00 & 30.00 & 30.00 & 30.00 \\
\hline $\begin{array}{l}\text { \% Año escolar en no } \\
\text { Presencialidad Óptima }\end{array}$ & 0.48 & 0.48 & 0.48 & 0.48 & 0.48 \\
\hline $\begin{array}{l}\text { Efectividad componente } \\
\text { no presencial }\end{array}$ & 0.16 & 0.24 & 0.29 & 0.39 & 0.50 \\
\hline $\begin{array}{l}\text { Pérdidas de aprendizaje por } \\
\text { Cierre }\end{array}$ & 12.07 & 10.90 & 10.17 & 8.76 & 7.14 \\
\hline $\begin{array}{l}\text { Elasticidad ingreso } \\
\text { inasistencia escolar }\end{array}$ & 0.90 & 0.90 & 0.90 & 0.90 & 0.90 \\
\hline $\begin{array}{l}\text { Disminución del ingreso } \\
\text { del hogar }\end{array}$ & 0.05 & 0.05 & 0.05 & 0.05 & 0.05 \\
\hline $\begin{array}{l}\text { Pérdidas de aprendizaje } \\
\text { inasistencia por pérdida } \\
\text { Ingreso }\end{array}$ & 1.35 & 1.35 & 1.35 & 1.35 & 1.35 \\
\hline Pérdida total de aprendizaje & 13.42 & 12.25 & 11.52 & 10.11 & 8.49 \\
\hline $\begin{array}{l}\text { Pérdida de aprendizaje } \\
\text { como \% año escolar }\end{array}$ & 0.45 & 0.41 & 0.38 & 0.34 & 0.28 \\
\hline $\begin{array}{l}\text { Años de escolaridad } \\
\text { esperado de } 4 \text { a } 18 \text { años }\end{array}$ & 11.30 & 11.30 & 11.30 & 11.30 & 11.30 \\
\hline $\begin{array}{l}\text { Años de escolaridad } \\
\text { ajustados por aprendizaje }\end{array}$ & 6.30 & 6.30 & 6.30 & 6.30 & 6.30 \\
\hline $\begin{array}{l}\text { Años de escolaridad sin } \\
\text { optimización modalidades }\end{array}$ & 5.85 & 5.89 & 5.92 & 5.96 & 6.02 \\
\hline $\begin{array}{c}\text { Ganancias salariales en } \\
\text { US\$ corriente }\end{array}$ & $4,117.33$ & $4,117.33$ & $4,117.33$ & $4,117.33$ & $4,117.33$ \\
\hline $\begin{array}{l}\text { Pérdidas por alumno por } \\
\text { reducción aprendizaje, US\$ }\end{array}$ & 147.31 & 134.51 & 126.44 & 110.95 & 93.24 \\
\hline $\begin{array}{c}\text { Valor presente pérdidas de } \\
\text { escolaridad por alumno, } \\
\text { US\$ }\end{array}$ & $2,618.39$ & $2,390.76$ & $2,247.44$ & $1,972.04$ & $1,657.30$ \\
\hline $\begin{array}{c}\text { Pérdida Sistema Educativo } \\
\text { no universitario, MM de } \\
\text { US\$ }\end{array}$ & $6,284.12$ & $5,737.83$ & $5,393.86$ & $4,732.91$ & $3,977.53$ \\
\hline Pérdida como \% del PIB & $7.99 \%$ & $7.29 \%$ & $6.85 \%$ & $6.01 \%$ & $5.05 \%$ \\
\hline $\begin{array}{l}\text { Pérdida como \% del } \\
\text { Presupuesto MINERD }\end{array}$ & $31.9 \%$ & $29.2 \%$ & $27.4 \%$ & $24.1 \%$ & $20.2 \%$ \\
\hline
\end{tabular}

Fuente: elaboración propia. 
Las cifras anteriores reflejan que con el cierre de los centros educativos en un $80 \%$, el promedio de las pérdidas de aprendizaje de todos los medios no presenciales utilizados representa el $10.53 \%$ del PIB, mientras que con la presencialidad de la combinación óptima de ambas modalidades, las pérdidas promedio se reducen a $6.64 \%$ del PIB, lo que significa que con la decisión pura y simple del cierre, sin tomar en cuenta las posibilidades de combinaciones virtualidad-presencialidad, que minimizan el costo educativo total del Covid 19, las autoridades educativas pierden la oportunidad de ahorrar el $3.89 \%$ del PIB en pérdidas por reducción de aprendizaje por exceso de la modalidad no presencial.

\section{Conclusión fundamental: reducir la desigualdad y aumentar la eficiencia en el logro de aprendizaje con medios no presenciales son los grandes retos de los sistemas educativos}

El análisis anterior demuestra que quienes más soportarán la carga de las pérdidas del aprendizaje serán los alumnos con menos accesos a los medios no presenciales eficientes, es decir, los más pobres de ingresos o en términos multidimensionales. Los grandes impactos de la crisis pandémica sobre la desigualdad en la distribución de sus costos educativos solo se pueden reducir de dos formas:

Primero, minimizando la suma del costo pandémico educativo total, lo cual requiere que en los centros donde el costo del contagio es muy bajo, se utilice la modalidad presencial de manera preponderante, y viceversa en los centros urbanos de alta densidad de alumnos y de población urbana.

Segundo, aumentando la eficiencia (mediante tutorías, ayudas personalizadas, centros comunitarios) de los medios no presenciales menos efectivos, como son los casos de la radio y la televisión, cuya efectividad por sí sola sería extremadamente escasa. 


\section{Bibliografía}

Bettinger E., Fairlie R. W., Kapuza A., Kardanova E., Loyalka P., Zakharov A. (2020). Does Edtech Substitute for Traditional Learning? Experimental Estimates of the Educational Production Function. NBER Working Paper. National Burean of Economic Research, (26967). Recuperado de: https:// publications.hse.ru/en/preprints/357673279.

Cattaneo, M., Oggenfuss C., y Wolter, S. C. (2016). The More, the Better? The Impact of Instructional Time on Student Performance. IZA DP, (9797) Recuperado de http://ftp.iza.org/dp9797.pdf.

Education Endowment Foundation (2019). Digital Technology Moderate Impact for Moderate Cost, Based on Extensive Evidence. https://educationendowmentfoundation.org.uk/evidence-summaries/teachinglearning-toolkit/digital-technology.

Escueta, M., Quan, V., Nickow, A. J. \& Oreopoulos, P. 2017. "Education Technology: An Evidence-Based Review.” NBER Working Paper w23744.

Filmer, Deon, Halsey Rogers, Noam Angrist and Shwetlena Sabarwal. Forthcoming. Learning Adjusted Years of Schooling (LAYS): Defining a New Macro Measure of Education. Economics of Education Review. Disponible en: https://doi.org/10.1016/j. econedurev.2020.101971.

Muralidharan, K., Singh, A. \& Ganimian, A. J. (2019). Disrupting Education? Experimental Evidence on Technology-Aided Instruction in India. American Economic Review, 109(4), 1426-1460.

Perera, M. \& Aboal, D, 2019. "The impact of a mathematics computer-assisted learning platform on students' mathematics test scores," MERIT Working Papers 2019-007, United Nations University - Maastricht Economic and Social Research Institute on Innovation and Technology (MERIT). Recuperado de https://ideas.repec. org/p/unm/ unumer/2019007.html

Reyes, R. (2020) Modelo de minimización de los costos totales de la cuarentena y la pandemia: aplicaciones y estimaciones para el caso de República Dominicana. Ciencia, Economía y Negocios, 4(2), 75-85.

Singh, A. (2019). Learning More with Every Year: School Year Productivity and International Learning Divergence. Journal of the European Economic Association. Doi: 10.1093/jeea/jvz033 
World Bank Group. (June 2020). Simulating the Potential Impacts of Covid-19 School Closures on Schooling and Learning Outcomes: A Set of Global Estimates. Conference Edition.

Zmmiskey, C., \& Stern, J. (2020). Calculating the Educational Impact of COVID-19 (Part II): Using Data from Successive Grades to Estimate Learning Loss. Recuperado de: https://shared.rti.org/content/calculating-educational-impact-covid-19-part-ii-using-data-successive-grades-estimate 